\title{
AVALIAÇÃO DA PREVALÊNCIA E DO PERFIL DE SENSIBILIDADE DE STAPHYLOCOCCUS COAGULASE NEGATIVA RESISTENTES À METICILINA/OXACILINA NO HOSPITAL UNIVERSITÁRIO DE SANTA MARIA (HUSM) E COMPARAÇÃO DE TESTES PARA DETECÇÃO DE RESISTÊNCIA
}

\author{
Evaluation of the prevalence and sensitivity profile of methicillin/oxacillin resistant coagulase negative \\ Staphylococci in the Hospital Universitário de Santa Maria (HUSM) and comparison \\ of tests for detection of resistance
}

Letícia Eichstaedt Mayer', Rosmari Horner ${ }^{2}$

\begin{abstract}
RESUMO
Os estafilococos coagulase negativa (ECN) têm sua patogenicidade cada vez mais reconhecida. Além das infecções relacionadas aos dispositivos implantados, participam como agentes etiológicos em diversas infecções. A emergência de cepas resistentes à meticilina/oxacilina reduz as opções terapêuticas, pois são resistentes a todos os â-lactâmicos e às combinações de â-lactâmicos/inibidores de â-lactamase. Nesse contexto, diversos métodos têm sido empregados para a detecção da resistência à meticilina/oxacilina em ECN. No presente estudo avaliou-se 50 isolados de ECN resistentes à oxacilina. Staphylococcus epidermidis foi a espécie mais isolada $30(60 \%)$, sendo o sangue o espécime clínico prevalente $19(38 \%)$ e a pediatria a unidade com o maior percentual de isolamentos $14(28 \%)$. Estas cepas apresentaram elevado perfil de resistência sendo que $62 \%$ delas mostraram-se sensíveis apenas à vancomicina, teicoplanina e tigeciclina. O método "screening" com ágar oxacilina detectou a resistência em todos isolados. Os discos de oxacilina 1 ìg e cefoxitina 30 ìg não detectaram somente em dois isolados cada.

Palavras-chave: estafilococos coagulase negativa; resistência oxacilina/meticilina; detecção de resistência

SUMMARY

Coagulase-negative staphylococci (CNS) have their pathogenicity increasingly recognized. Apart from infections related to implanted devices, they are etiological agents involved in various infections. The emergence of methicillin/oxacillin resistant CNS strains reduces treatment options, because they are resistant to all â-lactams and combinations of â-lactams/ â-lactamase inhibitors. In this context, several methods have been employed to detect methicillin/oxacillin resistance in CNS. In this study we evaluated 50 isolates of oxacillin-resistant CNS. Staphylococcus epidermidis was the species most often isolated $(30,60 \%)$, blood being the prevalent clinical specimen $(19,38 \%)$, and the pediatric unit with the highest percentage of isolates $(14,28 \%)$. These strains showed a high resistance profile and $62 \%$ of them were sensitive only to vancomycin, teicoplanin and tigecycline. The agar screening method detected oxacillin resistance in all isolates, and the disks of $1 \mathrm{mg}$ oxacillin and cefoxitin 30 ig failed to detect it only in two isolates each.

Keywords: Coagulase negative staphylococci, oxacillin resistance/methicillin, detection of resistance
\end{abstract}

\section{INTRODUÇÃO}

Os estafilococos coagulase-negativa (ECN) constituem um dos microrganismos mais comumente isolados no laboratório de bacteriologia. Fazem parte da flora normal da pele e mucosas e em certas ocasiões podem ser somente contaminantes em uma amostra. Porém, não há dúvida de que eles causam infecções severas, principalmente em pacientes hospitalizados. Por isso, as infecções freqüentemente resultam da introdução desses microrganismos em locais previamente estéreis, após traumas, abrasão da pele ou mucosas ou durante procedimento cirúrgico. A ocorrência de infecções nosocomiais causadas por

Trabalho realizado no Departamento de Análises Clínicas e Toxicológicas da Universidade Federal de Santa Maria - UFSM

${ }^{1}$ Acadêmica do Curso de farmácia do Centro de Ciências da Saúde da UFSM

${ }^{2}$ Professora Adjunta - Disciplina de microbiologia Clínica - Centro de Ciências da Saúde - Departamento de Análises Clínicas e Toxicológicas 
estafilococos é considerada um importante problema de saúde pública pelo número restrito de antimicrobianos efetivos no seu tratamento. A avaliação da sensibilidade a agentes antimicrobianos em isolados hospitalares e comunitários tem contribuído para o entendimento da distribuição e transmissão da resistência e ajudado na seleção da terapia antimicrobiana apropriada ${ }^{3}$.

Dentre os ECN, os mais freqüentes são Staphylococcus epidermidis seguido por Staphylococcus haemolyticus. Alguns trabalhos citam o $S$. epidermidis como a espécie prevalente nas bacteriemias, representando $74 \%$ a $92 \%$ dos isolamentos dos pacientes com processos infecciosos adquiridos no ambiente hospitalar. OS. haemolyticus tem sido associado a várias infecções como endocardites em válvulas naturais, septicemias, peritonites, infecções do trato urinário, feridas, infecções nos ossos e $\operatorname{articulações~}^{4,5,8}$.

Os ECNs vêm apresentando taxas elevadas de resistência à oxacilina, que variam de $66 \%$ a $93 \%$, principalmente em hospitais terciários e/ou de ensino. Por este motivo, atualmente são reconhecidos como microrganismos essencialmente oportunistas, que se provalecem de inúmeras situações orgânicas para produzir infecções graves ${ }^{2,3,4,5,6,7}$.

Vários métodos de detecção da resistência à oxacilina têm sido estudados, já que a expressão fenotípica desta resistência é usualmente heterogênea. A difusão com disco de oxacilina 1 ìg tem sido o método tradicionalmente utilizado na rotina no "screening" da resistência à meticilina/oxacilina neste grupo de bactérias. Porém, recentemente, a boa acurácia do disco de cefoxitina 30 ìg para a detecção da resistência à meticilina/oxacilina tem sido reportada. Assim, o Clinical and Laboratory Standards Institute/ National Comittee for Clinical Laboratory Standards (CLSI/ NCCLS), tem recomendado que o teste com disco de cefoxitina seja o método de preferência para testar ECN, pois possui especificidade maior e sensibilidade equivalente ao teste do disco de oxacilina, além de ser um forte indutor de sistema regulatório $\operatorname{mec}^{8,9,10,11,12}$

Os estafilococos são resistentes à oxacilina por três mecanismos: produção exagerada de â-lactamase, modificação das proteínas ligadoras de penicilinas (PBPs), presentes na parede celular desses microrganismos e pela presença de uma nova proteína na parede celular denominada penicillin-binding protein (proteína ligadora de penicilina) 2a, ou 2' (PBP2a ou PBP2'). Este último mecanismo é o mais importante nas cepas que são rotineiramente isoladas no laboratório. $\mathrm{O}$ gene mecA é o responsável pela síntese da PBP2a e a resistência fenotípica à oxacilina é extremamente variável dependendo da expressão desse gene. As cepas que têm esse mecanismo apresentam uma heterogeneidade dentro da colônia. Isto significa que uma porcentagem muito baixa dos clones dentro da colônia são resistentes enquanto que o resto da população é sensível. Isso ocorre porque todas as células carregam o gene mecA, marcador genotípico, porém nem todas expressam fenotipicamente sua resistência da mesma forma. É muito importante detectar de forma eficiente os microrganismos resistentes dentro dessa população causadora da infecção porque se o paciente é tratado com oxacilina, as cepas resistentes irão persistir no sítio da infecção ${ }^{1,8,11,13}$

O CLSI recomenda a diferenciação entre $S$. aureus e ECN, o que pode ser feito pelo teste da coagulase. Entre os ECN este instituto recomenda que seja identificado o $S$. saprophyticus em isolados da urina e não efetuar o antibiograma porque as infecções respondem a concentrações alcançadas na urina pelos agentes antimicrobianos normalmente usados para tratar infecções agudas, não complicadas do trato urinário (ex., sulfametoxazol-trimetoprim, nitrofurantoína ou uma fluorquinolona). Também se deve identificar outro SCN, o $S$. lugdunensis, um patógeno incomum, mas que pode causar endocardite. Um teste simplório para esta diferenciação é a pirrolidonil-â-naftilamida (PYR) - ( S. lugdunensis é $\mathrm{PYR}+)^{8,9}$. Também é necessário recordar que todas as cepas de estafilococos que são resistentes à oxacilina devem ser informadas como resistentes a todos os â-lactâmicos e às combinações de â-lactâmicos com inibidores de âlactamases $^{1,8,9}$

Nesse estudo, avaliou-se a prevalência, os perfis de sensibilidade e o desempenho dos discos de cefoxitina 30ì g, oxacilina 1̀̀g e o ágar screening oxacilina para a detecção de ECN resistentes à oxacilina em cinqüenta (50) cepas isoladas do Hospital Universitário de Santa Maria (HUSM).

\section{MATERIAIS E MÉTODOS}

Foram analisadas 50 (cinqüenta) amostras de ECN resistentes à meticilina/oxacilina coletadas prospectivamente e consecutivamente no Laboratório de Análises Clínicas do Hospital Universitário de Santa Maria (LAC-HUSM) no período de janeiro a maio de 2008. Os isolados foram provenientes de pacientes hospitalizados, de amostras clínicas diversas, tais como sangue, liquor, urina, ponta de cateter, ferida operatória, entre outros. No LAC do HUSM as amostras foram identificadas utilizando-se a automação (MicroScan DADE - Behring $($ ). O Teste de Sensibilidade Antimicrobiana (TSA) foi realizado através do painel para Gram-positivos (automação) e pelo método de disco difusão (Kirby-Bauer), com a disposição dos discos facilitando a visualização de possível achatamento de halo entre clindamicina e azitromicina, permitindo, assim a detecção de resistência induzível à clindamicina (Teste D).

A Tabela 1 mostra a listagem dos antimicrobianos testados e o grupo ao qual fazem parte, segundo CLSI 2008: grupo A (primeira escolha, testados e reportados na rotina); grupo $\mathrm{B}$ 
(primeira escolha, testados e reportados seletivamente, agentes importantes principalmente em infecções nosocomiais); grupo C (suplementares e reportados seletivamente, agentes alternativos quando ocorrem cepas resistentes a várias drogas primárias); grupo $\mathrm{U}$ ( testados em isolados de urina); grupo $\mathrm{O}$ (outros não rotineiramente utilizados) e grupo Inv. (em investigação).

Tabela 1 - Listagem dos Antimicrobianos testados neste estudo com as metodologias Automação e Kirby-Bauer e os respectivos grupos a que os antimicrobianos fazem parte

\begin{tabular}{llll}
\hline Metodologia Automação & & \multicolumn{2}{c}{ Metodologia Kirby-Bauer } \\
Antimicrobiano & Grupo & Antimicrobiano & Grupo \\
\hline Amoxicilina/Ácido Clavulânico & $\mathrm{O}$ & Azitromicina & $\mathrm{A}$ \\
Ampicilina/Sulbactam & $\mathrm{O}$ & Clindamicina & $\mathrm{A}$ \\
Ampicilina & $\mathrm{O}$ & Teicoplanina & Inv. \\
Cefazolina & $\mathrm{O}$ & Tigeciclina & $(*)$ \\
Ceftriaxona & $\mathrm{O}$ & Vancomicina & $\mathrm{B}$ \\
Ciprofloxacina & $\mathrm{C}$ & Oxacilina & $\mathrm{A}$ \\
Clindamicina & $\mathrm{A}$ & Cefoxitina & $(* *)$ \\
Eritromicina & $\mathrm{A}$ & & \\
Gentamicina & $\mathrm{C}$ & & \\
Levofloxacina & $\mathrm{C}$ & & \\
Nitrofurantoína & $\mathrm{U}$ & & \\
Norfloxacino & $\mathrm{U}$ & & \\
Oxacilina & $\mathrm{A}$ & & \\
Penicilina & $\mathrm{A}$ & & \\
Rifampicina & $\mathrm{B}$ & & \\
Sinercid & $\mathrm{C}$ & & \\
Sulfametoxazol/Trimetoprima & $\mathrm{A}$ & & \\
Vancomicina & $\mathrm{B}$ & & \\
\hline & & & \\
& & & \\
\end{tabular}

* O antimicrobiano tigeciclina não possui halo na listagem do CLSI (2008); foi aprovada pelo Food And Drug Administration (FDA) em junho de $2005^{14}$

** Cefoxitina é agente antimicrobiano para predição de resistência dos estafilococos mediada pelo mecA. O teste com disco de cefoxitina $30 \mathrm{igg} \mathrm{tem} \mathrm{a} \mathrm{finalidade} \mathrm{de} \mathrm{detectar} \mathrm{a} \mathrm{resistência}$ à oxacilina e não à própria cefoxitina. Conforme o diâmetro devese relatar como resistente ou sensível à oxacilina ${ }^{9,11}$.

Após este "screening" as cepas de ECN foram encaminhadas ao Laboratório de Bacteriologia do Departamento de Análises Clínicas e Toxicológicas (DACT) do Centro de Ciências da Saúde (CCS), prédio 26, sala 1201, onde se procedeu à identificação convencional através da coloração de Gram, teste da catalase e teste da coagulase em tubo. As cepas foram armazenadas em TSB (Tryptone-Soy Broth) $15 \%$ de glicerol, à $-20^{\circ} \mathrm{C}$.

Os isolados foram analisados, através dos testes com disco de cefoxitina 30ìg, disco de oxacilina 1ìg em placas de ágar Mueller Hinton, inoculadas com uma suspensão equivalente à escala 0,5 de McFarland do ECN isolado clínico. As placas foram incubadas a $35^{\circ} \mathrm{C}$ por $24 \mathrm{~h}$ e as zonas de inibição de crescimento foram medidas e classificadas em Sensíveis e Resistentes segundo os tamanhos dos halos referidos pelo CLSI 2008.

Procedeu-se também ao teste com ágar oxacilina Probac ${ }^{\circledR}$ (ágar Mueller Hinton com 4\% de $\mathrm{NaCl}$ e 6ìg de oxacilina por $\mathrm{mL}$ ), meio de triagem de resistência, no qual inoculou-se uma suspensão equivalente a escala 0,5 de McFarland do ECN isolado clínico. Incubou-se a $35^{\circ} \mathrm{C}$ por $24 / 48 \mathrm{hs}$; qualquer crescimento foi interpretado como resultado positivo para ECN, isto é, reportado como resistente à oxacilina/meticilina.

Todos os testes foram realizados em duplicata e o controle de qualidade dos discos foi feito utilizando-se a cepa ATCC n ${ }^{\circ} 25923$ de S. aureus.

\section{RESULTADOS}

Dos 50 (cinqüenta) isolados analisados neste estudo, pode-se estabelecer VI (seis) perfis de sensibilidade a partir dos testes de disco-difusão, o que está representado na Tabela 2 .

O teste de screening com ágar oxacilina mostrou-se positivo para todos os isolados testados. Não foi necessária a incubação por $48 \mathrm{hs}$, pois todos os isolados apresentaram crescimento em 24horas.

A positividade encontrada para o Teste D foi de $6(12 \%)$ dos isolados, e destes, 5 (10\%) apresentaram o perfil I de resistência, ou seja, mostraram-se sensíveis apenas à vancomicina, teicoplanina e tigeciclina. 
Tabela 2 - Perfis de Sensibilidade dos 50 (cinqüenta) isolados de ECN no LAC HUSM no período de janeiro a maio de 2008 pelo método de difusão em disco

\begin{tabular}{lllllllll}
\hline PERFIL & VC & TEC & TGC & CLI & AZI & CFO & OXA & $\mathrm{n}(\%)$ \\
\hline I & S & S & S & R & R & R & R & $31(62)$ \\
II & S & S & S & S & S & R & R & $10(20)$ \\
III & S & S & S & S & R & R & R & $6(12)$ \\
IV & S & S & S & R & R & R & S & $1(2)$ \\
V & S & S & S & S & S & S & R & $1(2)$ \\
VI & S & S & S & S & S & S & S & $1(2)$ \\
Total & & & & & & & & $50(100)$ \\
\hline
\end{tabular}

$\mathrm{VC}=$ vancomicina $. \mathrm{TEC}=$ teicoplanina $. \mathrm{TGC}=$ tigeciclina. $\mathrm{CLI}=$ clindamicina $. \mathrm{AZI}=$ azitromicina $. \mathrm{CFO}=$ cefoxitina. $\mathrm{OXA}=$ oxacilina.

A Tabela 3 demonstra a distribuição conforme as espécies isoladas neste estudo. A identificação foi obtida pelo sistema de automação (MicroScan - DADE - Behring $\left.{ }^{\circledR}\right)$.

Tabela 3 - Distribuição dos 50 ECN isolados de pacientes atendidos no HUSM no período de janeiro a maio de 2008 de acordo com as espécies isoladas

\begin{tabular}{lcc}
\hline Espécie & Número de Amostras (n) & Percentual (\%) \\
\hline Staphylococcus epidermidis & 30 & 60 \\
Staphylococcus sp. & 14 & 28 \\
Staphylococcus haemolyticus & 5 & 10 \\
Staphylococcus saprophyticus & 1 & 2 \\
Total & 50 & 100 \\
\hline
\end{tabular}

O Gráfico 1 a seguir mostra a distribuição dos 50 isolados de ECN provenientes de pacientes atendidos no HUSM no período de janeiro a maio de 2008 , avaliados conforme o espécime clínico.

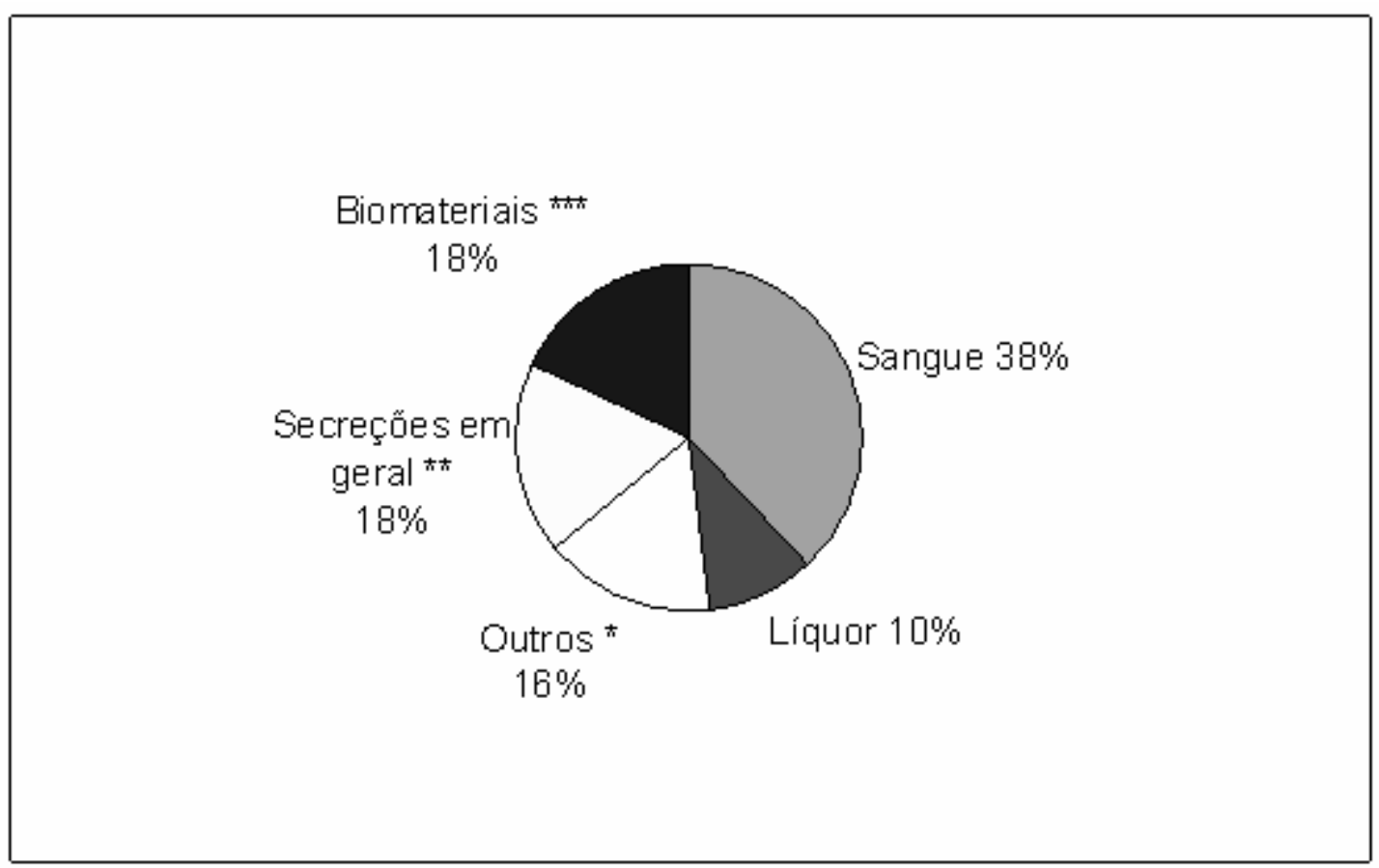

Gráfico 1 - Percentual de ECN isolados de acordo com o Espécime Clínico 
* Outros: urina, escarro, líquido diálise, lavado broncoalveolar.

** Secreções em geral: feridas operatórias, escara sacral, abscessos.

*** Biomateriais: cateteres, cânulas/tubos orotraqueais, pontas de drenos.

A distribuição dos isolados de ECN resistentes à oxacilina/meticilina em relação às unidades hospitalares pode ser observada na Tabela 4.

Tabela 4 - Distribuição dos 50 (cinqüenta) isolados ECN provenientes de pacientes atendidos no HUSM no período de janeiro a maio de 2008 de acordo com as Unidades Hospitalares

\begin{tabular}{ll}
\hline \multicolumn{1}{c}{ Unidades Hospitalares } & n (\%) \\
\hline Pediatria (= 13anos) (UTI-RN, UTI - pediátrica, Pronto Atendimento Pediátrico, Setor de Pediatria) & $14(28)$ \\
Pronto Atendimento Adulto & $7(14)$ \\
UTI Adulto & $7(14)$ \\
Centro de Transplante de Medula Óssea & $4(8)$ \\
Nefrologia & $4(8)$ \\
CTcriac - Centro de Tratamento de Crianças com Câncer (isolados provenientes de pacientes maiores & $4(8)$ \\
de 13 anos) & $10(20)$ \\
Outros (ambulatório, bloco cirúrgico, centro obstétrico, cirurgia, hematologia, cardiologia) & $50(100)$ \\
TOTAL & \\
\hline
\end{tabular}

A Tabela 5 mostra a relação entre unidade hospitalar de origem, espécime clínico, idade e a espécie identificada de 5 (cinco) pacientes $(\mathrm{A}, \mathrm{B}, \mathrm{C}, \mathrm{D}$ e E) que tiveram isolamento de $\mathrm{ECN}$ resistente à oxacilina/meticilina em mais de um material biológico.

Tabela 5 - Pacientes com isolamento, em mais de um material biológico, de ECN resistentes à oxacilina/meticilina

\begin{tabular}{lllll}
\hline PACIENTE & IDADE & Espécime Clínico & Espécie Identificada * & Unidade \\
\hline A & 58 anos & Sangue & Staphylococcus sp. & PA \\
& & Líquor & Staphylococcus sp. & adulto \\
B & recém-nascido & Cânula Orotraqueal & S. epidermidis & UTI - \\
& & Sangue & Staphylococcus sp. & RN \\
C & recém-nascido & PICC & S. epidermidis & PA \\
& & Sangue & S. epidermidis & adulto \\
D & \multirow{2}{*}{ anos } & Escarro & S. epidermidis & UTI \\
& & Sangue & Staphylococcus sp. & CTMO \\
& & & & S. epidermidis \\
\hline
\end{tabular}

* Espécie Identificada através do Sistema Automatizado (MicroScan - DADE-Behring®).

$\mathrm{PA}=$ Pronto Atendimento. $\quad \mathrm{PICC}=$ Cateter Central de Inserção Periférica.

$\mathrm{CTMO}=$ Centro de Transplante de Medula Óssea.

Os perfis de sensibilidade dos ECN resistentes à oxacilina/meticilina, frente a alguns antimicrobianos, obtidos a partir do painel para Gram-positivos utilizado na automação - MicroScan® estão representados na Tabela 6. 
Tabela 6 - Perfis de sensibilidade aos antimicrobianos dos 50 (cinqüenta) isolados de ECN resistentes à oxacilina/meticilina obtidos de pacientes atendidos no HUSM, no período de janeiro a maio de 2008 utilizando-se a automação (MicroScan ${ }^{\circledR}$ )

\begin{tabular}{lcccc}
\hline Antimicrobianos (Grupo) & Sensíveis (\%) & Intermediários (\%) & Resistentes (\%) & Total(\%) \\
\hline Ciprofloxacina (C) & 43 & - & 57 & 100 \\
Clindamicina (A) & 32 & 2 & 66 & 100 \\
Eritromicina (A) & 22 & - & 78 & 100 \\
Gentamicina (C) & 29 & - & 71 & 100 \\
Levofloxacina (C) & 41 & 24 & 35 & 100 \\
Rifampicina (B) & 67 & 2 & 2 & 100 \\
Sinercid (C) & 91,5 & 6,5 & 53 & 100 \\
Sulfameoxazol/Trimetoprima (A) & 47 & - & - & 100 \\
Vancomicina (B) & 100 & - & & 100 \\
\hline
\end{tabular}

\section{DISCUSSÃO}

Observando-se a Tabela 2 observa-se que um percentual elevado $31(62 \%)$ de isolados mostrou-se sensível apenas aos antimicrobianos vancomicina, teicoplanina e tigeciclina. Os perfis IV, V e VI merecem atenção especial: no perfil IV, um isolado apresentou-se sensível à oxacilina; no perfil $\mathrm{V}$, um isolado mostrouse sensível à cefoxitina. Este fato concorda com os dados da literatura, em que se cita a inclusão (e não substituição) do disco de cefoxitina, e prediz-se que nenhum método é isoladamente adequado ${ }^{13,15}$. Ou seja, a utilização apenas do disco cefoxitina, ou apenas do disco oxacilina não prediz em todos os casos a resistência à oxacilina/meticilina. Além disso, no perfil VI, observouse um isolado sensível para os testes de disco-difusão, tanto com disco de cefoxitina quanto oxacilina, mas detectou-se a resistência através do "screening" ágar oxacilina.

A espécie $S$. epidermidis foi a prevalente $(60 \%$ dos isolamentos) dado que se encontra de acordo com outros estudos no Brasil $^{4,5,8,16,17}$ (Tabela 3). O único Staphylococcus saprophyticus foi isolado de urina, fato explicado por essa bactéria apresentar uma aderência preferencial às células esfoliativas do trato urogenital quando comparada a sua habilidade em aderir às células de outros setores como pele e boca ${ }^{18}$. Dos $5(10 \%)$ isolados de $S$. haemolyticus, 3 (6\%) foram provenientes de feridas em geral.

O sangue apresentou-se como o espécime clínico com maior número de isolados representando 19 (38\%) isolamentos (Gráfico I). Este fato corrobora com os dados da literatura em que os ECN são isolados prevalentes em septicemias, peritonites, infecções do trato urinário, feridas, materiais implantáveis (biomateriais), entre outros $4,5,8,16,18,19,20,21$.

Nesse estudo evidenciamos 14 isolados (28\%) de ECN resistentes à oxacilina/meticilina provenientes de pacientes com idade inferior ou igual a 13 anos (Tabela 4) e 9 (18\%) destes isolados eram pacientes pediátricos nos quais foi encontrado o perfil I (conforme Tabela 2), ou seja, apresentaram elevada resistência aos antimicrobianos testados, mostrandose sensíveis apenas à vancomicina, teicoplanina e tigeciclina. Vários estudos têm avaliado a significância clínica das infecções por ECN em pacientes pediátricos, revelando grande incidência de bacteremia hospitalar verdadeira, particularmente nos recém-nascidos. Nesse contexto, revelam-se necessários estudos dirigidos para essas populações, bem como concentrar a vigilância epidemiológica no setor de pediatria, visando ao controle e ou implementação de medidas de controle efetivas $^{5,19,22}$

Nesse estudo, verificamos que cinco pacientes (A, B, C, $\mathrm{D}$ e E) apresentaram isolados de ECN resistentes à oxacilina em mais de um material biológico (Tabela 5); em três(3) deles, paciente $\mathrm{B}, \mathrm{C}$ e E, observa-se que o biomaterial pode ter sido o responsável como porta de entrada para o microrganismo, levando à hemocultura positiva, ou seja, disseminação do processo infeccioso. Além disso, trata-se de pacientes críticos ( recém-nascido e de transplante de medula óssea).

Em relação à suscetibilidade aos antimicrobianos testados por técnica automatizada, (Tabela 6), todos os isolados testados apresentaram-se sensíveis à vancomicina; $91,5 \%$ mostraram-se sensíveis ao sinercid (combinação de estreptograminas A e B) e $67 \%$, sensíveis à rifampicina. $\mathrm{O}$ antimicrobiano que apresentou maior taxa de resistência foi eritromicina (78\%), seguido por gentamicina $(71 \%)$ e 
clindamicina $(66 \%)$.

\section{CONCLUSÕES}

- os testes com disco de cefoxitina 30ìg e oxacilina lìg devem ser usados concomitantemente para atuarem de forma complementar na deteç̧ão de resistência à oxacilina/meticilina;

- o teste de "screening" com ágar oxacilina mostrou desempenho excelente, detectando a resistência em todos os isolados testados. Sugere-se a inclusão do teste de screening com ágar oxacilina na rotina laboratorial para detecção de resistência à oxacilina/meticilina em $\mathrm{ECN}$, além de fácil execução, pode-se testar vários isolados ao mesmo tempo, apesar de nenhuma recomendação pelo CLSI/2008;

- a espécie prevalente nos isolados testados foi $\mathrm{S}$. epidermidis (60\%);

- o sangue foi o espécime clínico com o maior número de isolados, representando $38 \%$;

- $28 \%$ dos isolados foram provenientes de pacientes pediátricos ( $\leq 13$ anos de idade) e destes, grande parte apresentou elevada resistência, mostrando-se sensíveis apenas para os antimicrobianos vancomicina, teicoplanina e tigeciclina.

- 91,5\% dos isolados mostraram-se sensíveis ao sinercid;

- eritromicina, gentamicina e clindamicina foram os antimicrobianos que apresentaram maiores taxas de resistência, $78 \%, 71 \%$ e $66 \%$, respectivamente;

- todos os isolados foram sensíveis à vancomicina;

\section{REFERÊNCIAS BIBLIOGRÁFICAS}

1- Palavecino RE. Métodos recomendados para el estudio de susceptibilidad en Staphylococcus aureus, Staphylococcus coagulasa negativa y Staphylococcus saprophyticus: Nuevos puntos de corte e interpretación de resultados. Rev. chil. infectol. [online]. 2002, vol.19 supl.2 [citado 31 Março 2008], p.119-124. Disponível na World Wide Web: http://www.scielo.cl/scielo.php?script= sci_arttext\&pid $=$ S0716$10182002019200011 \& \operatorname{lng}=p t \& n r m=i s o>$. ISSN 0716-1018.

2- Blatt JM; Piazza CE. Perfil de sensibilidade de cepas Staphylococcus aureus e Staphylococcus coagulase negativo isolados em pacientes internados. Revista Brasileira de Análises Clínicas 6(2):129- 131, 2004.

3- Michelim et al. Pathogenicity factors and antimicrobial resistance of Staphylococcus epidermidis associated with nosocomial infections occurring in intensive care units. Braz. J. Microbiol. , São Paulo, v. 36, n. 1, 2005 . Disponível em: <http:/ /www.scielo.br/scielo.php? script=sci_arttext \&pid=S1517$83822005000100004 \& \operatorname{lng}=\mathrm{en} \& n r m=\mathrm{iso}>$. Acesso em: $31 \mathrm{Mar}$ 2008. doi: $10.1590 / \mathrm{S} 1517$-83822005000100004

4- Hörner R et al. Perfil da Suscetibilidade dos microrganismos isolados de uma unidade de terapia intensiva neonatal. Saúde, Santa Maria, vol.32, n1, p 15-21, 2006.

5- Cunha MLRS et al. Significância clínica de estafilococos coagulase-negativa isoaldos de recém-nascidos. Jornal de Pediatria, Rio de Janeiro, v. 78, n. 4, p. 279-288, 2002.

6- Menezes EA et al. Frequêencia e percentual de suscetibilidade de bactérias isoladas em pacientes atendidos na Unidade de Terapia Intensiva do Hospital Geral de Fortaleza - J Bras Patol Med Lab -v. $43 \cdot$ n. $3 \cdot$ p. $149-155 \cdot$ junho 2007

7- Quesada RMB et al. Culturas de pontas de cateteres venosos centrais e perfil de resistência aos antimicrobianos de uso clínico. Revista Brasileira de Análises Clínicas, v. 37, n. 1, p. 45-48, 2005.

8- Terasawa LB. Caracterização da resistência à oxacilina em estafilococos coagulase negativa isolados no hospital de clínicas de Curitiba - Paraná. 2006. Dissertação (mestrado) - Setor de Ciências Biológicas e da Saúde. Programa de Pós-Graduação em Ciencias Biológicas (Microbiologia, Parasitologia e Patologia Básica). Universidade Federal do Paraná, Curitiba.

9- Clinical and Laboratory Standards Institute. Performance Standards for Antimicrobial Susceptibility Testing; Fiftwenth Informational Supplement. CLSI document M100-S18 (ISBN 1-156238-556-9). Wayne, Pensylvania, 4sA, 2008.

10- Frigatto EAM et al. Is the cefoxitin disk test reliable enough to detect oxacillin resistance in coagulase-negative staphylococci? Journal of Clinical Microbiology, v. 43, n. 4, p. 2028-2029, 2005.

11- Felten A et al. 2002. Evaluation of three techniques for detection of low-level methicillin-resistant Staphylococcus aureus (MRSA): a disk diffusion method with cefoxitin and moxalactam, the Vitek 2 system, and the MRSA-screen latex agglutination test. J. Clin. Microbiol. 40:2766-2771.

12- Okonogi $\mathrm{K}$ et al. 1989. Emergence of methicillin-resistant clones from cephamycin-resistant Staphylococcus aureus. J. Antimicrob. Chemother. 24:637-645.

13- Mimica MJ; Mendes CMF. Diagnóstico laboratorial da resistência à oxacilina em Staphylococcus aureus. Artigo de Revisão. J Bras Patol Med Lab. V.43 n.6 p.399-406. Dezembro 2007.

14- Food and Drugadministration - FDA - Disponível em: $<$ http://www.fda.gov/ medwatch/safety/2006/Jul_PIs/ Tygacil_PI.pdf $>$. Acesso em: 07 jul.2008

15- Ricardo, Silvana de Barros. Problemas no diagnóstico laboratorial dos padrões de resistência aos antimicrobianos. $1^{\circ}$ Encontro em Pneumonia Hospitalar: o desafio do MRSA. Águas de São Pedro (Publicação Seriada). Outubro, 2006. Parte 2:8-10. 16- Leão LSNO et al. Fenotipagem de bactérias isoladas em hemoculturas de pacientes críticos. Revista da Sociedade Brasileira de Medicina Tropical, 40(5):537-540, set-out, 2007. 17- Alcaráz LE et al. Species identification, slime production and oxacillin susceptibility in coagulase-negative staphylococci isolated from nosocomial specimens. Brazilian Journal of 
Microbiology (2003) 34:45-51

18- Keim LS. Mapeamento dos Estafilococos Coagulase Negativo no Hospital Universitário Antônio Pedro da Universidade Federal Fluminense, no período de 1998 a 2002. 2005. Dissertação (Mestrado - Curso de Pós-Graduação em Ciências Médicas) - Universidade Federal Fluminense, Niterói, 2005.

19- Góngora-Rubio F et al. Significância clínica, epidemiologia e microbiologia das bacteremias por estafilococos coagulase-negativos em Hospital de Ensino. Rev Ass Med Brasil 1997; 43(1):9-14.

20- Stepanovic $S$ et al. A modified microtiter-plate test for quantification of staphylococcal biofilm formation. Journal of Microbiological Methods 40 (2000) 175-179

21 - Bernardi ACA. Estudo de Amostras de Staphylococcus coagulase-negativa quanto a formação de biofilme. 2005. Tese (Doutorado em Análises Clínicas - Área de Microbiologia Clínica) - UNESP, Araraquara, São Paulo, 2005.

22 - Felix JR LF. Infecção por Staphylococcus aureus resistente à oxacilina: Revisão da Literatura. 2007. Residência Médica em Pediatria. Centro de Estudos do Hospital Regional Asa Sul Brasília - DF, 2007.32p

Endereço para correspondência:

Rosmari Hörner

rosmari.ufsm@gmail.com 